\title{
SOLUBILIZATION OF P-ALKYLPHENOLS IN PLURONICS F-68 AND F-127 MICELLES: PARTITION COEFFICIENTS AND EFFECT OF SOLUTE ON THE AGGREGATE STRUCTURE
}

\author{
ANDRÉS F. OLEA ${ }^{I^{*}}$, HÉCTOR CARRASCO ${ }^{1}$, LUIS ESPINOZA ${ }^{2}$, BETSABE ACEVEDO

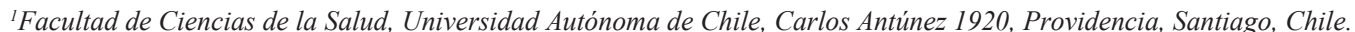 \\ ${ }^{2}$ Departamento de Química, Universidad Técnica Federico Santa María, Av. España No. 1680, Valparaíso 2340000, Chile. \\ ${ }^{3}$ Departamento de Ciencias Químicas, Facultad de Ciencias Exactas, Universidad Andrés Bello, Quillota 910, Viña del Mar, Chile.
}

\begin{abstract}
The partition of a series of substituted phenols between water and polymer micelles formed by Pluronic F- 68 and F-127 has been studied. The formation of micelles by these block copolymers has been evidenced by steady-state fluorescence and dynamic light scattering. The data show that micelles and larger aggregates are formed above the critical micelle concentration. In presence of p-alkylphenols a micelle rearrangement occurs that leads to the formation of just one and large aggregate. This effect depends both on polymer and phenols structures. The partition coefficients and the standard free energies of transfer from the aqueous to the micellar phases were determined. The incremental free energy for a methylene group are $2.88 \mathrm{~kJ} \mathrm{~mol}^{-1}$ and $1.65 \mathrm{~kJ} \mathrm{~mol}^{-1}$ for F-127 and F- 68 , respectively. This difference is explained in terms of the core sizes that are determined by the length of the PPO block.
\end{abstract}

Keywords: Pluronic, block copolymers, polymer micelles, partition, free energy of transfer, critical micelle concentration, fluorescence probing methods, dynamic light scattering.

\section{INTRODUCTION}

In the recent decade much effort has been dedicated to the development of nanoscale materials that can be used as drug delivery systems (DDS) ${ }^{1-3}$. To date these materials include polymer-drug conjugates, polymer-protein conjugates, and polymeric micelles within which the drugs can be simply entrapped or covalently bound ${ }^{4}$. Most of polymeric micelles, used as DDS, are core-shell structures where the hydrophobic segments form the core of the micelle while the hydrophilic part forms the corona or outer shell. A number of natural and synthetic polymers with linear, branched, and dendritic structures have been studied. However, most of the studies of drug solubilization have been carried out with amphiphilic block copolymers ${ }^{5-7}$. Examples of such kind of polymers are A-B-A triblock copolymers known by the trade name of Pluronic, which consist of a central hydrophobic block of poly(propylene oxide) (PPO) and hydrophilic end blocks of poly(ethylene oxide) (PEO), PEO-PPO-PEO. These copolymers are commercially available in a range of molecular weights and $\mathrm{PPO} / \mathrm{PEO}$ composition ratios. In aqueous solution at concentrations above the critical micelle concentration (CMC) Pluronics form micelles having spherical, cylindrical, lamellar or vesicular morphologies. The CMC depends on temperature and critical micellar temperatures (CMT) have been measured. Above the CMT, the micellar size generally increases with increasing temperature before the solution phase separates at the cloud point (CP). On approaching the CP many of these copolymer solutions exhibit sphere to rod transitions ${ }^{8}$. From all the Pluronics, F-127 has shown to be a very interesting one owing to its potential use as DDS ${ }^{9-11}$, removal of aromatic contaminants from industrial waste waters ${ }^{12,13}$, and in preparation of mesoporous materials ${ }^{14-16}$. Pluronic F-127 form spherical micelles where the core of the micelles consists of hydrophobic PPO blocks, whereas the corona is formed by hydrated PEO blocks ${ }^{17}$. The properties and potential application of these micelles as DDS has been studied by several groups ${ }^{8,9,18,19}$. The suitability of these polymers as drug carriers depends on key micelle properties, such as stability, loading capacity, and release kinetics. The loading capacity of polymeric micelles is related to the size and size distribution of the aggregates, and to the partition coefficient that measure the extent of incorporation into the micelles. To date the studies where the partition coefficients were determined are scarce ${ }^{20,21}$, and there are relatively few studies concerning the effects of high molecular weight solutes and drugs on the structure of polymer micelles 22,23 .

In the present work the solubilization of a series of p-alkylphenol derivatives into polymeric micelles formed by Pluronic F-68 and F-127 has been investigated. The partition coefficients between the aqueous and micellar phases were determined by using the pseudo-phase model. The results are compared with previous work where these model compounds have been used to study normal micelles and intramolecular polymer micelles ${ }^{24-27}$. In addition, the effect of incorporation of phenols on the micelle structure has been assessed.

2. Experimental Part

2.1 Materials. Pluronic F-68 and F-127 (Aldrich) and p-alkylphenols (Aldrich) were used without further purification. Aqueous solutions of
Pluronics (0.001M in a molar chain unit basis) and p-alkylphenols $(0.1 \mathrm{M})$ were prepared with distilled and deionized water obtained from an EASYpure RF (Barnstead) deionization system. Samples for fluorescence measurements were prepared by adding a concentrated solution of pyrene in acetonitrile to aqueous solutions of Pluronics.

2.2 Dynamic light scattering. The size distributions of polymeric micelles were determined by dynamic light scattering (DLS). Aqueous solutions of Pluronics $(1 \mathrm{mM})$ in absence and presence of p-alkylphenols $(10 \mathrm{mM})$ were thermostated at $25{ }^{\circ} \mathrm{C}$ and DLS measurements were performed using a Zetasizer Nano ZS (Malvern Instruments, Malvern, UK) equipped with a $\mathrm{He}-$ $\mathrm{Ne}$ laser (633 nm, $4 \mathrm{~mW})$.

2.3 Fluorescence probing. Steady-state fluorescence spectra of pyrene were recorded utilizing a Horiba Jobin Yvon Fluoromax 4 fluorometer by exciting at $337 \mathrm{~nm}$. All emission spectra were corrected for detector response using a correction curve supplied by the instrument manufacturer. The ratio I / $\mathrm{I}_{3}$ corresponds to the ratio of intensities of peak one $(\lambda=372 \mathrm{~nm})$ to peak three $(\lambda=384 \mathrm{~nm})$, whereas the ratio $I_{M} / I_{E}$ corresponds to the ratio of intensities of the monomer band to the excimer band $(\lambda=450 \mathrm{~nm})$.

2.4 Measurement of distribution coefficients. In the pseudo-phase model the solubilization process is represented by the equilibrium

$$
\mathrm{S}_{\mathrm{W}}+\mathrm{P}_{\mathrm{M}} \rightleftarrows \mathrm{S}_{\mathrm{M}}
$$

For which the equilibrium constant Ks is given by

$$
K_{S}=\frac{[S]_{M}}{[S]_{W}[P]_{M}}
$$

where $[\mathrm{S}]_{\mathrm{W}}$ and $[\mathrm{S}]_{\mathrm{M}}$ denote molar concentration of substrate in aqueous and in the polymeric pseudo-phase, respectively; $[\mathrm{P}]_{\mathrm{M}}$ is the concentration of polymer chains forming the hydrophobic aggregates. On a mole fraction basis the partition coefficient can be expressed by

$$
K_{X}=\frac{X_{M}}{X_{W}}
$$

where $X_{M}$ and $X_{W}$ are the mole fraction of substrate in the micellar and aqueous phases, respectively. In diluted solutions, both quantities are related by

$$
\mathrm{K}_{\mathrm{X}}=55.5 \mathrm{~K}_{\mathrm{S}}
$$

The distribution coefficients (Ks) were obtained by ultrafiltration using an Amicon 202 cell with a PM5 membrane, which has a MW cut-off of 5,000. Aqueous solutions of Pluronic $(0.001 \mathrm{M}$ in a chain unit basis) containing 
different concentrations of p-alkylphenols were filtered, and the absorbances of the filtrate were measured at $275 \mathrm{~nm}$. The molar concentrations of phenols were obtained from a calibration curve. The values of $\mathrm{K}_{\mathrm{S}}$ were obtained from the slope in a plot of $\left[\mathrm{S}_{\mathrm{M}}\right]$ against $\left[\mathrm{S}_{\mathrm{W}}\right]$.

\section{RESULTS AND DISCUSSION}

3.1 Characterization of aggregates formed by Pluronics. Two Pluronics copolymers, F-68 and F-127 have been used in this study. These triblock copolymers differ both in molecular weights and $\mathrm{PPO} / \mathrm{PEO}$ composition ratio. The percentage of ethylene oxide is similar in both copolymers but F-127 has a highest molecular weight. The structural parameters of Pluronics F-68 and F-127 are shown in table 1.

Table 1. Structural parameters of Pluronics and critical micelle concentration.

\begin{tabular}{|c|c|c|c|c|c|}
\hline Pluronic & $\mathrm{Mw}$ & PPO units & PEO units & $\%$ PEO & $\mathrm{CMC}^{\mathrm{a}}, \mathrm{M}$ \\
\hline F-68 & 8400 & 29 & 153 & 80 & $4.4 \times 10^{-4}$ \\
\hline F-127 & 12600 & 65 & 200 & 70 & $2.7 \times 10^{-6}$ \\
\hline
\end{tabular}

${ }^{a} \mathrm{CMC}$ values were determined by using pyrene as fluorescence probe.

It is well established that F-68 and F-127 copolymers self-aggregate in aqueous solution forming spherical micelles above a critical micelle concentration ${ }^{17}$. In this work the values of $\mathrm{CMC}$ were determined by measuring the ratio $\mathrm{I}_{\mathrm{M}} / \mathrm{I}_{\mathrm{E}}$ of pyrene fluorescence as a function of Pluronic concentration. The results given in Table 1 are similar to those found in literature ${ }^{28}$. The sizes and size distributions of aggregates formed by F-68 and F-127 were obtained from DLS measurements (Figure 1). It is worth to mention that DLS provides the micelle hydrodynamic average diameter, which includes the water molecules present in the corona. Interestingly, the presence of three different aggregates is observed, i.e. spherical micelles with average sizes of $5 \mathrm{~nm}$ and $7 \mathrm{~nm}$ for F-68 and F-127, respectively, and large micelles clusters whose average sizes range from 38 to $460 \mathrm{~nm}$ (see Table 2). The average sizes of micelles agree well with data from literature, and the coexistence of micelles and larger aggregates has also been observed in other Pluronics solutions ${ }^{29,30}$. These results reveal the formation of small spherical micelles along with larger micellar clusters in proportion that depends on the polymer structure. For both copolymers the most intense signals of scattered light correspond to small spherical micelles, indicating that these have the highest relative abundance.

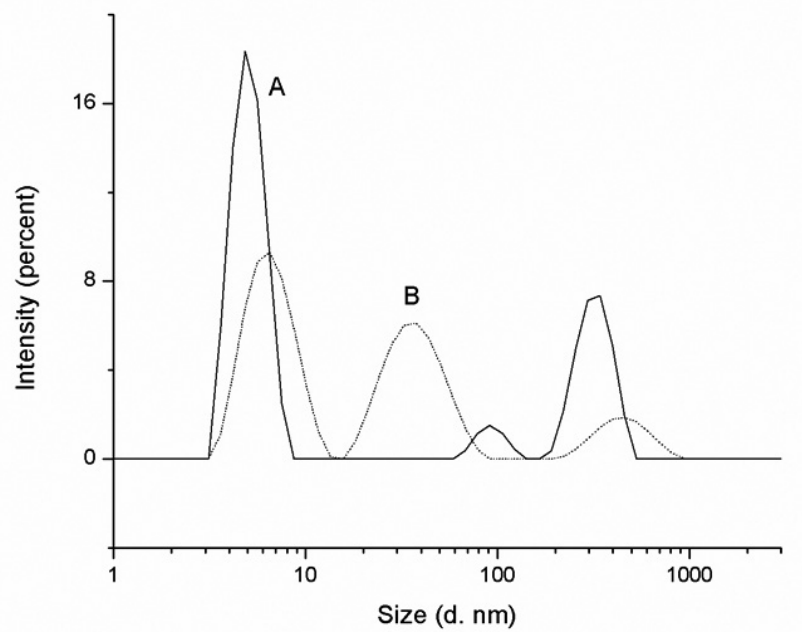

Figure 1. Distribution sizes of aggregates formed by Pluronic F-68 (A) and F-127 (B). DLS measurements were made at $25^{\circ} \mathrm{C}$ with Pluronic concentration equal to $1 \mathrm{mM}$.

3.2 Effect of p-alkylphenol on size distribution of Pluronics micelles. The effect of the addition of p-alkylphenols on the structure of the aggregates formed by Pluronic F-68 and F-127 has been investigated by DLS. The results shown in Figures 2 and 3 indicate clearly that in all cases the size distribution changes in the presence of phenols. For both Pluronics this effect depends on the polymer and phenol structure. In the case of F-127 the incorporation of phenols brings about the complete disappearance of the peaks associated to small micelles and large aggregates, and the appearance of a new peak centered at $29 \mathrm{~nm}$. In other words, the incorporation of phenols induces a rearrangement of the aggregates that leads to the formation of a unique aggregate of average diameter equal to $29 \mathrm{~nm}$. However, this rearrangement process is partial in presence of p-cresol. On the other hand, in F-68 solutions p-alkylphenols produce a gradual disappearance of small micelles, and only slight changes in the sizes of large aggregates are observed. The exception is p-butylphenol that exhibits an effect similar to p-cresol on F-127.

A micelle growth has been observed by solubilization of phenol and other additives in the micelles formed by F-127 $7^{31,32}$. It has been postulated that the incorporated solutes replaces water molecules associated to the PEO units and consequently the micelle hydrophobicity is increased. This effect of micellar dehydration enhances intermicellar attractive interaction and therefore the formation of large micellar clusters ${ }^{32}$.

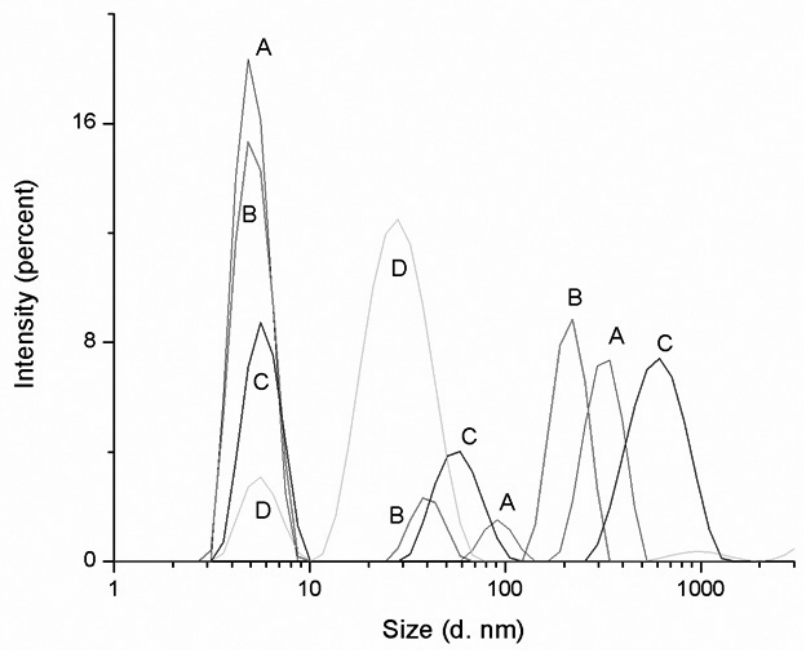

Figure 2. Distribution sizes of aggregates formed by Pluronic F-68 (1mM) in absence (A) and presence of p-ethylphenol (B), p-propylphenol (C) and p-butylphenol (D). DLS measurements were made at $25^{\circ} \mathrm{C}$. Concentration of phenols is $10 \mathrm{mM}$.

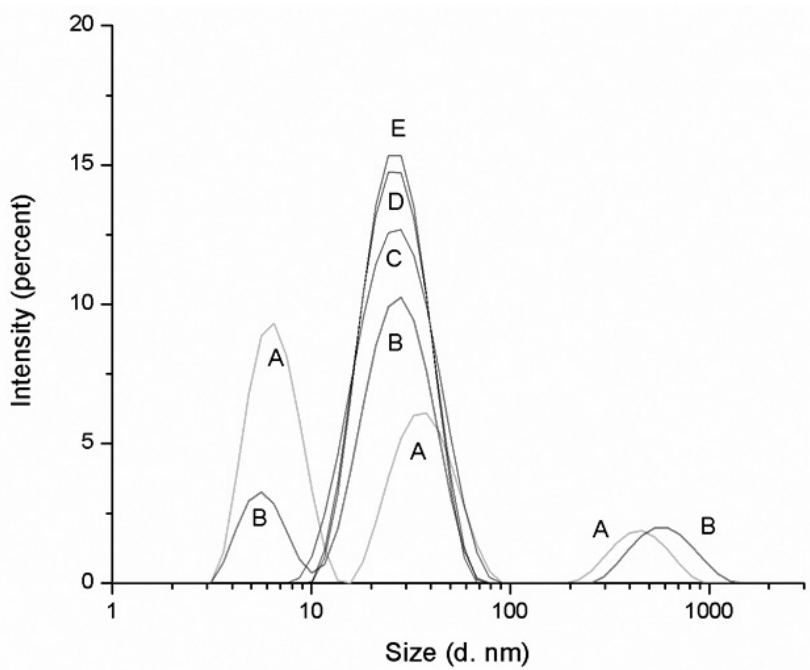

Figure 3. Distribution sizes of aggregates formed by Pluronic F-127 $(1 \mathrm{mM})$ in absence (A) and presence of p-cresol (B), p-ethylphenol (C), p-propylphenol (D) and p-butylphenol (E). DLS measurements were made at $25^{\circ} \mathrm{C}$. Concentration of phenols is $10 \mathrm{mM}$.

In order to verify the dehydration effect of phenols the variation of micelle micropolarity has been monitored by fluorescence probing using the ratio $I_{1} / I_{3}$ 
of pyrene fluorescence. It has been shown that this ratio is related to the polarity of the medium where pyrene is located ${ }^{33,34}$. Thus, this parameter has been used to study micelles and other aggregates that provide hydrophobic environments in aqueous solution ${ }^{35-37}$. In polar media this ratio takes high values (in aqueous solution $\mathrm{I}_{1} / \mathrm{I}_{3}=1.92$ ) and it is shown to decrease with decreasing polarity. The values of this ratio measured in aqueous solution of Pluronics $(1 \mathrm{mM})$ in absence and presence of p-alkylphenols $(10 \mathrm{mM})$ are given in Table 2. In solutions of F-68 and F-127 the values of $\mathrm{I}_{1} / \mathrm{I}_{3}$ are 1.76 and 1.56 , respectively, indicating that the micropolarity of micelle core is higher in F-68 than in F-127 micelles. In presence of substituted phenols, for both Pluronics, the ratio $\mathrm{I}_{1} /$ $\mathrm{I}_{3}$ decreases with increasing length of the phenol alkyl chain. In the case of $\mathrm{F}-127, \mathrm{I}_{1} / \mathrm{I}_{3}$ diminishes continuously reaching a value of 1.31 in presence of p-butylphenol, whereas for F-68 the ratio changes slightly, excepting for p-butylphenol $\left(\mathrm{I}_{1} / \mathrm{I}_{3}=1.25\right)$ that is the only molecule inducing an important growth of micellar size.

Table 2. Micellar sizes and ratio $I_{1} / I_{3}$ of pyrene fluorescence measured in aqueous solutions of Pluronics $(1 \mathrm{mM})$ and p-alkylphenols $(10 \mathrm{mM})$. Pyrene concentration is $0.2 \mu \mathrm{M}$.

\begin{tabular}{|c|c|c|c|c|}
\hline & \multicolumn{2}{|c|}{ Pluronic F-127 } & \multicolumn{2}{c|}{ Pluronic F-68 } \\
\hline p-Alkylphenol & Diameter, nm & $\mathrm{I}_{1} / \mathrm{I}_{3}$ & Diameter, nm & $\mathrm{I}_{1} / \mathrm{I}_{3}$ \\
\hline None & $7 ; 38 ; 461$ & 1.56 & $5 ; 93 ; 322$ & 1.76 \\
\hline p-cresol & $6 ; 29 ; 614$ & 1.54 & $5 ; 78 ; 547$ & 1.74 \\
\hline p-ethylphenol & 28 & 1.37 & $5 ; 40 ; 215$ & 1.73 \\
\hline p-propylphenol & 28 & 1.32 & $6 ; 58 ; 624$ & 1.64 \\
\hline p-butylphenol & 28 & 1.31 & $6 ; 30$ & 1.25 \\
\hline
\end{tabular}

Thus, in presence of p-alkylphenols the polarity of aggregates decreases with increasing hydrophobicity of phenols. The effect of phenol structure could be explained in terms of the partition coefficient of these compounds between the micellar and aqueous phases. Usually, the phenol concentrations in the micellar phase are higher for the more hydrophobic compounds, and therefore their effects on micelle structure are larger. On the other hand, it has been shown that the partition coefficient of non polar molecules increases with increasing PPO content and molecular weight. Comparing the structures of F-68 and F-127 (Table 1) it is seen that these two conditions should favor the partition of phenols to F-127. The partition coefficients are determined and discussed below.

In conclusion, the change in size distribution can be attributed to two simultaneous effects, i.e. incorporation of additives and variation in the aggregation number. In other words, the incorporation of non polar substrates increases the core volume and, at the same time, by the dehydrating effect increases the aggregation number of the small micelles. These structural changes lead to disappearance of small micelles and formation of a larger aggregate. However, as most of the phenols molecules are incorporated into small micelles (because they have the higher relative abundance) the disappearance of the larger aggregates existing in pure solutions of Pluronics (see Figure 1) should have another explanation. A possibility is to consider different states of aggregation that are in equilibrium, as it is assumed in the closed association model. In this model, the molecularly dispersed polymer chains (unimer) are in equilibrium with larger aggregates. Thus, the diminution in the number of small micelles induces a decrease in the number of large aggregates that eventually disappear.

3.3 Partition of p-alkylphenols between the aqueous and micellar phases. The distribution coefficients of phenols between the micellar and aqueous phases, $\mathrm{K}_{\mathrm{s}}$, defined by Eq. (2) were determined for F-68 and F-127. From these equilibrium constants the partition coefficient in a mole fraction basis, $\mathrm{K}_{\mathrm{x}}$ was calculated by using Eq. (3). The results are summarized in Table 3.

Table 3. Partition coefficients and standard free energies of transfer of p-alkylphenols between aqueous phase and micelles formed by Pluronics

\begin{tabular}{|c|c|c|c|c|}
\hline & \multicolumn{2}{|c|}{ Pluronic F-127 } & \multicolumn{2}{c|}{ Pluronic F-68 } \\
\hline p-Alkylphenol & $\mathrm{K}_{\mathrm{x}}, \times 10^{4}$ & $\begin{array}{c}\Delta \mu_{\mathrm{t}}^{0}, \mathrm{~kJ} \\
\mathrm{~mol}^{-1}\end{array}$ & $\mathrm{~K}_{\mathrm{x}}, \times 10^{4}$ & $\Delta \mu_{\mathrm{t}}^{0}, \mathrm{~kJ} \mathrm{~mol}^{-1}$ \\
\hline p-cresol & 1.8 & 24.23 & 2.0 & 24.50 \\
\hline p-ethylphenol & 8.9 & 28.20 & 2.8 & 25.40 \\
\hline p-propylphenol & 16.1 & 29.70 & 9.8 & 28.45 \\
\hline p-butylphenol & 70.5 & 33.45 & 12.2 & 29.00 \\
\hline
\end{tabular}

As expected the partition coefficients increases with length of alkyl chain, and the values of $\mathrm{K}_{\mathrm{x}}$ are larger for F-127 micelles than F-68 micelles. These results agree with data obtained for the partition of phenols in micelles, unimolecular and intermolecular polymer micelles ${ }^{24-26,38}$ Also, the effect of Pluronic structure on the values of $\mathrm{K}_{\mathrm{x}}$ follows the trend observed for the solubilization of other non polar substrates, i.e. the partition coefficient increases with increasing PPO content and molecular weight, F-127 $>$ F- $68^{20}$.

The standard free energy of transfer of phenols from the aqueous phase to the micellar phase is expressed as

$$
\Delta \mu_{t}^{0}=\mu_{M}^{0}-\mu_{W}^{0}=-R T \ln K_{X}
$$

It has been proposed that $\Delta \mu^{0}$ can be separated in additive contributions from different groups present in the solute ${ }^{24}$

$$
\Delta \mu_{t}^{0}=\Delta \mu_{A r}^{0}+n_{C} \Delta \mu_{C}^{0}
$$

where $\Delta \mu^{0}{ }_{\text {Ar }}$ denotes the contribution of the parent aromatic group, $\Delta \mu_{C}^{0}$ is the incremental free energy per methylene group, and $n_{C}$ is the number of these groups attached to the parent group. Similar linear free energy relationships have been found in studies of partition of solutes between aqueous phase and organic solvents, micelles, and polymer micelles ${ }^{24,25,39-41}$. In Figure 4 the values of $\Delta \mu_{\mathrm{t}}^{0}$ are plotted against the number of carbon atom in the p-alkylphenol.

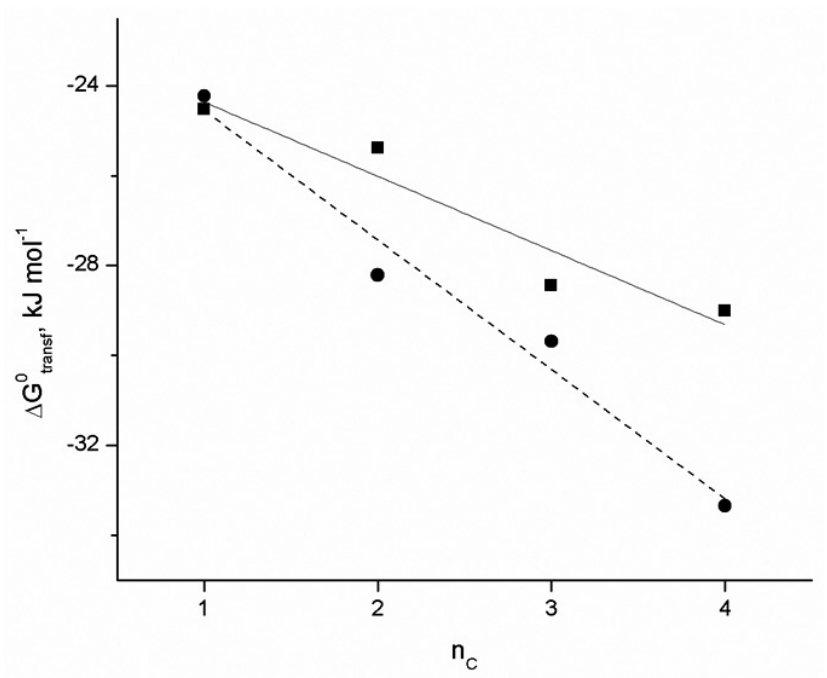

Figure 4. Standard free energies of transfer of phenols from aqueous to micellar phase as a function of the number of carbon atoms in the p-alkyl chain: $(\bullet)$ F-127; (•) F-68.

The intercepts obtained from these plots are -21.7 and $-22.7 \mathrm{~kJ} \mathrm{~mol}^{-1}$ for F-127 and F-68, respectively. These values are quite similar and the difference is in the error range. On the other hand, the values of the incremental free energy per methylene group obtained from the slopes are $2.88 \mathrm{~kJ} \mathrm{~mol}^{-1}$ and $1.65 \mathrm{~kJ} \mathrm{~mol}^{-1}$ for F-127 and F-68, respectively. The value obtained for F-127 agrees well with the one determined by measuring the partition coefficients of a family of n-alkylfluorescein $\left(2.55 \mathrm{~kJ} \mathrm{~mol}^{-1}\right)^{28}$. However, both values of $\Delta \mu^{0}{ }_{\mathrm{c}}$ are lower than that determined for transfer of methylene group to heptane $(3.27$ $\left.\mathrm{kJ} \mathrm{mol}^{-1}\right)^{42}$, suggesting that the cores of Pluronic micelles are less hydrophobic than typical non polar solvents. This conclusion is on line with relative micropolarity of micelles determined by the ratio $\mathrm{I}_{1} / \mathrm{I}_{3}$ (see Table 2 ).

The data also indicate that the values of $\Delta \mu^{0}{ }_{C}$ depend on the structure of Pluronic copolymer, i.e. the incremental free energy increases with increasing length of PPO block. In comparison with F-127 the PPO block of F-68 is two times shorter, and therefore in F-68 micelles the core is smaller as well. Consequently, solutes dissolved into the F-68 micelle core experience steric hindrance and a more polar environment. As a result the incremental free energy of transfer in F-68 micelles is lower than in F-127 micelles. 


\section{CONCLUSIONS}

The results of this study concerning the solubilization of a family of p-alkylphenols in micelles formed by Pluronic copolymers demonstrate the existence of an important effect of solute incorporation on the micelle structure. DLS measurements of micelles formed by F-68 and F-127 show the existence of micelles and larger aggregates. The size distribution is completely changed by the solubilization of phenols into the micelle core. In the case of F-127 the solute induces the formation of a stable aggregate with average diameter equal to $29 \mathrm{~nm}$. This growth is attributed to the simultaneous incorporation of phenol and increase in the aggregation number. The latter effect is attributed to a decrease in the micelle polarity produced by the replacement of water molecules of the corona, and it was verified by measuring the ratio $\mathrm{I}_{1} / \mathrm{I}_{3}$ of pyrene fluorescence. The dependence with polymer structure is rationalized in terms of the partition coefficient of these compounds. The values of $\mathrm{K}_{\mathrm{X}}$ were obtained by using the pseudo-phase model and the results show that the partition and incremental free energy are determined mainly by the length of the PPO block.

\section{ACKNOWLEDGMENTS}

The authors are grateful to FONDECYT for financial support of this work under Grants 1130742 and 1120996.

\section{REFERENCES}

1. M. E. Davis and M. E. Brewster Nat. Rev. Drug Discov., 3, 1023 (2004)

2. M. L. Adams, A. Lavasanifar, and G. S. Kwon J. Pharm. Sci., 92, 1343 (2003)

3. R. T. Chacko, J. Ventura, J. Zhuang, and S. Thayumanavan Adv. Drug Deliv. Rev., 64, 836 (2012)

4. R. Haag and F. Kratz Angew. Chem. Int. Ed., 45, 1198 (2006)

5. C. Allen, D. Maysinger, and A. Eisenberg Colloids Surf. , B., 16, 3 (1999)

6. K. Kataoka, A. Harada, and Y. Nagasaki Adv. Drug Deliv. Rev., 47, 113 (2001)

7. G. S. Kwon and K. Kataoka Adv. Drug Deliv. Rev., 16, 295 (1995)

8. P. Alexandridis and T. A. Hatton Colloids Surf. , A., 96, 1 (1995)

9. R. Basak and R. Bandyopadhyay Langmuir, 29, 4350 (2013)

10. Z. Sezgin, N. Yuksel, and T. Baykara Int. J. Pharm., 332, 161 (2007)

11. M. A. Sharp, C. Washington, and T. Cosgrove J. Colloid Interf. Sci., 344, $438(2010)$

12. Y. K. Choi, S. B. Lee, D. J. Lee, Y. Ishigami, and T. Kajiuchi J. Membr. Sci., 148, 185 (1998)

13. L. Heerema, D. Cakali, M. Roelands, E. Goetheer, D. Verdoes, and J. Keurentjes Sep Purif. Technol., 74, 55 (2010)

14. F. Zhang, Y. Meng, D. Gu, Y. Yan, Z. Chen, B. Tu, and D. Zhao Chem. Mat., 18, 5279 (2006)
15. M. Li and J. Xue J. Colloid Interf. Sci., 377, 169 (2012)

16. J. Schuster, R. Koehn, M. Doeblinger, A. Keilbach, H. Amenitsch, and T. Bein J. Am. Chem. Soc., 134, 11136 (2012)

17. R. Nagarajan Colloids Surf. , B., 16, 55 (1999)

18. A. V. Kabanov, E. V. Batrakova, and V. Y. Alakhov J. Control. Release, $82,189(2002)$

19. M. A. James-Smith, D. Shekhawat, B. M. Moudgil, and D. O. Shah Langmuir, 23, 1640 (2007)

20. P. N. Hurter and T. A. Hatton Langmuir, 8, 1291 (1992)

21. S. R. Croy and G. S. Kwon J. Control. Release, 95, 161 (2004)

22. P. K. Sharma and S. R. Bhatia Int. J. Pharm., 278, 361 (8-7-2004)

23. P. K. Sharma, M. J. Reilly, D. N. Jones, P. M. Robinson, and S. R. Bhatia Colloids Surf., B., 61, 53 (2008)

24. L. Sepulveda, E. A. Lissi, and F. Quina Adv. Colloid Interf. Sci., 25, 1 (1986)

25. A. F. Olea, R. G. Barraza, I. Fuentes, B. Acevedo, and F. Martinez Macromolecules, 35, 1049 (2002)

26. R. G. Barraza, A. F. Olea, C. E. Valdebenito, V. Dougnac, and I. Fuentes J. Colloid Interf. Sci., 275, 434 (2004)

27. A. F. Olea, in Ionic Interactions in Natural and Synthetic Macromolecules, A. Ciferri and A. Perico, Ed., John Wiley. 2012.

28. M. Y. Kozlov, N. S. Melik-Nubarov, E. V. Batrakova, and A. V. Kabanov Macromolecules, 33, 3305 (2000)

29. W. Brown, K. Schillén, M. Almgren, S. Hvidt, and A. Bahadur J. Phys. Chem., 95, 1850 (1991)

30. W. Brown, K. Schillén, and S. Hvidt J. Phys. Chem., 96, 6038 (1992)

31. R. Ganguly, K. Kuperkar, P. Parekh, V. Aswal, and P. Bahadur J. Colloid Interf. Sci., 378, 118 (2012)

32. J. Dey, S. Kumar, S. Nath, R. Ganguly, V. Aswal, and K. Ismail J. Colloid Interf. Sci., 415, 95 (2014)

33. K. Kalyanasundaram and J. K. Thomas J. Am. Chem. Soc., 99, 2039 (1977)

34. D. C. Dong and M. A. Winnik Photochem. Photobiol., 35, 17 (1982)

35. A. F. Olea and J. K. Thomas Macromolecules, 22, 1165 (1989)

36. A. F. Olea, B. Acevedo, and F. Martinez J. Phys. Chem. B, 103, 9306 (1999)

37. A. F. Olea, P. Silva, I. Fuentes, F. Martinez, and D. Worrall J. Photochem. Photobiol. A: Chem., 217, 49 (2011)

38. A. F. Olea and C. Gamboa J. Colloid Interf. Sci., 268, 63 (2003)

39. C. Tanford, The Hydrophobic Effect, John Wiley, New York, 1988

40. C. Gamboa, A. F. Olea, H. E. Rios, and M. Henriquez Langmuir, 8, 23 (1992)

41. C. H. Salamanca, R. G. Barraza, B. Acevedo, and A. F. Olea J. Chil. Chem. Soc., 52, 1025 (2007)

42. C. Hirose and L. Sepulveda J. Phys. Chem., 85, 3689 (1981). 